\title{
Efficacy of intratympanic \\ injection Dexamethasone and Triamcinolone on SSNHL
}

\author{
Mojtaba Meybodian, Mohsen Abouii*, Mohammadhossein Dadgarnia, Mohammadhossein \\ Baradaranfar, Sedighe Vaziribozorg
}

Department of Otolaryngology- Head and Neck Surgery, Otorhinolaryngology Research Center, Shahid Sadoughi University of Medical

Sciences, Yazd, Iran

*Corresponding Author: Mohsen Abouii, Department of Otolaryngology- Head and Neck Surgery, Otorhinolaryngology Research Center, Shahid Sadoughi University of Medical Sciences, Yazd, Iran

Email: m.abouii2020@gmail.com; Tel: 00983538224000

Received: June 10, 2020; Accepted: June 22, 2020

\begin{abstract}
Objective: The purpose of this study was to compare the effect of intratympanic injection of dexamethasone and triamcinolone on sudden sensorineural hearing loss (SSNHL). Methods: In this study 60 patients with SSNHL were divided into two groups of Dexamethasone 8 mg and Triamcinolone $40 \mathrm{mg}$. Patients in both groups underwent five injections. Hearing status of the two groups was recorded one day before each injection as well as one week and one month after the last injection at different frequencies. Any possible complications following the injection, such as infection and tympanic membrane perforation was evaluated and recorded. Results: The results showed that after the first and second injection, there was no significant difference between the two groups regarding hearing frequency and speech discrimination score (SDS). Significant differences were observed between the two groups in the third and fourth injections at a frequency of $500 \mathrm{~Hz}$ and also in the fifth injection at frequencies of 500,1000, and $2000 \mathrm{~Hz}$. Tympanic membrane perforation was observed in three patients in the dexamethasone group (P.value $>0.05$ ). Conclusion: In our study, the effect of triamcinolone was found to be significantly better than dexamethasone in the treatment of SSHNL at lower frequencies. The SDS was also higher in the triamcinolone group than dexamethasone. Based on our results, intratympanic injection of both triamcinolone and dexamethasone are safe and effective treatments for the treatment of SSNHL, but given its desirable medicinal properties, intratympanic injection of triamcinolone can be a safe and effective alternative for SSNHL treatment.
\end{abstract}

Keywords: Sudden sensorineural hearing loss, Triamcinolone, Dexamethasone, Intratympanic injection.

\section{INTRODUCTION}

Idiopathic sudden sensorineural hearing loss (SSNHL) is defined as hearing loss of $30 \mathrm{~dB}$ or greater over a period of 3 days and at least at three consecutive frequencies without any known cause. SSNHL is usually unilateral and may be accompanied by dizziness or tinnitus. Bilateral type of the disease is very rare (1). Hearing loss can occur suddenly or within hours. In many cases, no specific etiology can be identified for this disease and in this case, it is called idiopathic SSNHL. Sudden head trauma, immune system disorders, vascular disorders, some medications, meningioma, acoustic neuroma, viruses, purulent meningitis, syphilis, AIDS, MS, and Sarcoidosis are among the risk factors of SSNHL. A variety of treatments are suggested for SSNHL, among which corticosteroids usually play an important role in this regard (2). To treat this disease, systemic corticosteroid, is used, or it is directly injected into the middle ear. Intratympanic steroid treatment by direct injection and creating high medication concentration in the ear, while avoiding the side effects induced by systemic treatment, seems to be an appropriate option according to a large number of studies $(3,4)$. Intratympanic administration of dexamethasone, as one of the most important medicinal compounds in this field, is used to treat SSNHL. Triamcinolone is a synthetic glucocorticoid compound that is used because of its anti-inflammatory and long-lasting effects on various autoimmune diseases (5). There have been many studies on the use of dexamethasone in the treatment of SSNHL $(6-8)$. Since there have not been many studies on the efficacy of triamcinolone in treating SSNHL, the present study aimed to compare the efficacy of dexamethasone and triamcinolone in this regard. 


\section{MATERIALS AND METHODS}

After approval by the local ethics committee and obtaining written informed consent from all the patients, 60 patients aged 20-60 years who suffered from unilateral hearing loss (occurred within 2 hours) were included in this randomized clinical trial study. Mean of hearing threshold was greater than $30 \mathrm{~dB}$ in these patients, and onset of symptoms was reported within 7 days from initiation of the study. Patients with a history of middle ear disorder, autoimmune diseases, vestibular disorder (e.g. Ménière disease), cardiovascular diseases, prior ear surgery, and those treated with other treatments such as intravenous injection or systemic oral medication, were excluded. Demographic characteristics (age and sex), history of diseases such as hypertension and diabetes and symptoms associated with the disease (tinnitus and vertigo) were recorded. Patients were randomly divided into two groups using a random number table. Patients in group one received intratympanic injections of dexamethasone $(8 \mathrm{mg})$ and patients in the second group received intratympanic injections of triamcinolone $(40 \mathrm{mg})$. Patients in both groups underwent five injections (Injections were repeated every other day and in the middle ear.) Before injections, the volume of medication in the syringes was equalized, and the patient was not aware of the type of medication in the syringe. Hearing status of the two groups was recorded one day before each injection as well as one week and one month after the last injection using the audiometry and determining the hearing threshold at different frequencies of $500,1000,2000,4000$, and 8000 . All patients underwent otoscopic examination one week after the last injection and one month thereafter to identify any possible complications following the injection, such as infection and tympanic membrane perforation. Injections were continued to respond to treatment was achieved or until the 5th injection. The response to treatment criterion was defined (Based on AAO-HNS: American Academy of Otolaryngology-Head and Neck Surgery) as complete recovery from hearing loss less than $10 \mathrm{~dB}$ compared to the opposite ear or SDS recovery to less than five to $10 \%$ of the opposite ear). More than five injections were not done in the case of any response to treatment. The data were analyzed using SPSS (version 22) and running descriptive statistics, including mean (Mean of hearing frequency improvement and mean of speech discrimination score (SDS) between two groups and also mean of hearing frequency improvement before and after each injection was recorded) and variance and appropriate statistical tests, such as T-test (independent and paired) and repeated measure analysis. $\mathrm{P}$-value $<0.05$ was considered significant.

\section{RESULTS}

A total of 60 patients were included in this study based on our defined inclusion and exclusion criteria. There was no statistically significant difference regarding demographic variables of age ( $p$.value $=0.42)$, sex $(p$. value $=0.43)$, prevalence of diseases such as hypertension ( $p . v a l u e=0.56$ ) and diabetes (p.value $=0.36$ ) and also mean of the number of injections (p.value $=0.35$ ) and finally in symptoms associated with the disease ( $p$.value=0.14) between two groups. After the first injection, there was no significant difference between the two groups in mean of the hearing frequency improvement and mean speech discrimination score (SDS) (p.value>0.05). There was no significant difference between the two groups after the second injection either (p.value>0.05). After the third injection, no significant difference between the two groups was detected except only at a frequency of $500 \mathrm{~Hz}$, the observed difference in mean of the hearing frequency improvement was significant $(47.66 \pm 22.1$ in dexamethasone group versus $31.16 \pm 25$ in triamcinolone group (p.value=0.03). The two groups did not even differ significantly after the fourth injection except only at frequencies of 500 and $1000 \mathrm{~Hz}$, the observed differences in mean of the hearing frequency improvement were significant (p.value $=0.03$, p.value $=0.04$ respectively). After the fifth injection, a significant difference was observed in mean of the hearing frequency improvement between the two groups at the frequencies of 500, 1000, and $2000 \mathrm{~Hz}$ (p.value $=0.03$, p.value $=0.04$ and p.value $=0.03$ respectively). At other frequencies $(4000,8000)$, the observed difference in mean of the hearing frequency improvement and SDS was not significant (p.value>0.05) (Tables.1, 2, 3, 4, 5). Frequency improvements before and after each injection were also evaluated between the two groups and the significant effect of both treatments on all hearing frequencies was evident ( $p$.value $\leq 0.05$ ). Finally, there was no significant difference between two groups in tympanic membrane perforation (p.value $=0.237$ ) following injection.

\section{DISCUSSION}

SSNHL is one of the hearing losses disorders that can be very distressing to the patient because of its suddenness and severity. The rate of recovery in patients with untreated SSNHL was reported to be 31 to 65 percent (9), while the recovery rate in treated patients was 35 to 89 percent (10-12). These findings can be attributed to different factors, including data provided by the patient, type of steroid used, duration of treatment, severity of hearing loss, length of time between symptom onset and initiation of treatment, type of treatment protocol, and even method of data analysis (13). Despite all these differences, treatment with steroids has been proven in various studies. In this study, we compared the therapeutic efficacy of intratympanic injection of dexamethasone and triamcinolone in the treatment of SSNHL. According to the results of this study, both dexamethasone and triamcinolone significantly improved the hearing frequency at frequencies of 500, 1000, 2000, 4000, and $8000 \mathrm{~Hz}$. It was also found that triamcinolone was significantly better for treating SSNHL than dexamethasone and it had higher efficacy at higher frequencies. Similarly, Choung et al, found that intratympanic dexamethasone injection compared with systemic dexamethasone treatment led to significantly better hearing recovery in patients with SSNHL. Choung et al, found that intratympanic dexamethasone injection compared with systemic therapy would significantly improve recovery in patients with SSNHL(14). Their findings were in line with those found in the present study. In another study, Sung et al compared systemic and intratympanic steroid therapies. The time intervals between injections were also different in each group. The results of this study showed that the effect of intratympanic steroid injection was not significantly correlated with injection time intervals, and the rate of disease recovery in patients treated with daily intratympanic injection compared with patients treated intratympanic injection at intervals of two to three days was statistically the same (5). Our study also showed that intratympanic injection of both dexamethasone and triamcinolone, over a single day, significantly improved the hearing frequency and SDS in the patients. As noted, according to similar studies, the type of treatment protocol and the type of steroids used in treatment are effective in the rate of patient recovery (13). In our study, the type of used steroids resulted in a difference in the improvement of the hearing frequency in the final injections, so that in patients receiving triamcinolone, the recovery rate at frequencies of 500,1000 , and $2000 \mathrm{~Hz}$ was significantly better than those receiving dexamethasone. At 
other frequencies and also with respect to SDS, the triamcinolone group was better than the dexamethasone group, although the observed difference was not significant. So our study, along with other studies, showed the significant effect of the type of steroid in the treatment process of this disease. The precise mechanism of steroids in hearing improvement in SSNHL is still unknown. Glucocorticoid and mineralocorticoid receptors may be found in the inner ear. The main roles of steroids in the treatment of SSNHL include:

1. Protecting the cochlea from the harmful effects of inflammatory mediators, such as tumor necrosis factor (TNF- $\alpha$ and NF-kB) and cytokines (interleukins 1 and 6), which are highly involved in infection and inflammation (15),

2. Increasing cochlear blood flow and thus avoiding cochlear ischemia (16),

\section{Avoiding noise-induced hearing loss (17), and}

4. Regulating protein synthesis in the inner ear (18).

The greater efficacy of triamcinolone, compared with dexamethasone, in improving the disease our study may be due to the better effect of the medication on any of the abovementioned factors, especially the protection of cochlea from the harmful effects of inflammatory mediators. For the first time, Silverstein revealed that intratympanic steroid injection is effective in the treatment of sensorineural hearing loss, and subsequent other studies confirmed this finding $(19,20)$. These studies showed that intratympanic injection of steroids resulted in an increase in peripheral lymph node concentration compared to the systemic treatment. Our study also suggested the positive effect of intratympanic injection of both triamcinolone and dexamethasone. Most studies on the treatment of sensorineural hearing loss focused on using dexamethasone and methylprednisolone and there are few studies on the efficacy of triamcinolone in this regard. In another study, Dahm et al evaluated the effect of intratympanic triamcinolone and systemic prednisolone in patients with sensory-neurological hearing loss. The results of this study demonstrated that the treatment process was better in patients received both treatments, but there was no statistically significant difference between two groups. The aforementioned study also showed no significant difference between the two treatment modalities of prednisolone and triamcinolone regarding treatment process. They acknowledged that intratympanic administration of triamcinolone led to similar results compared to previous studies which used dexamethasone or methylprednisolone. Due to their favorable medicinal properties, they considered triamcinolone a safe and effective alternative for the treatment of sensory-neurological hearing loss (21). Our study, in line with their study, showed the better efficacy of triamcinolone than dexamethasone.
Commonly, intratympanic steroids are used for three main purposes, namely, (1) initial treatment, (2) adjuvant therapy that is co-administered with systemic steroids, and (3) subsequent treatment after failure in standard treatment. However, according to several clinical trials $(18,20,22)$, intratympanic injection of steroids, as the primary treatment, appear to be a valuable solution in the treatment of SSNHL, which is at least as effective as systemic steroids. In addition, it can be an appropriate option for patients who did not respond to intravenous treatment or cannot tolerate systemic therapy (23, 24). In line with these studies, our study also suggested the efficacy of treatment with intratympanic steroid injection in significantly improving hearing frequencies and SDS before and after the last injection. In our study, the two study groups were compared in terms of symptoms associated with the disease (no symptoms, tinnitus, and dizziness) as well as the effect of tympanic membrane perforation due to injections. Data analysis revealed no significant differences between groups in this regard. Similar studies also showed that the type of steroid had no significant effect on drug induced adverse effects. Suzuki et al (25), Ahn et al (26) and Ashtiani et al (27) also yielded that intratympanic triamcinolone and systemic dexamethasone had similar therapeutic effects and they were not significantly different in terms of adverse effects.

\section{CONCLUSION}

In our study, the effect of triamcinolone was found to be significantly better than dexamethasone in the treatment of SSHNL at lower frequencies. The SDS was also higher in the triamcinolone group than dexamethasone. On the other hand, the mean number of injections was lower in the triamcinolone group than in the dexamethasone group. Therefore, it seems that both intratympanic triamcinolone and dexamethasone are safe and effective for treatment of SSHNL but given its desirable medicinal properties, intratympanic injection of triamcinolone can be a safe and effective alternative for the treatment of SSHNL.

\section{"Compliance with Ethical Standards"}

* Authors have no conflicts of interest. * Study protocol was in accordance with the latest Declaration of Helsinki for medical research involving human subjects and was approved by the local ethics committee.

\footnotetext{
*This article does not contain any studies with animals performed by any of the authors.

* Informed consent was obtained from all participants of the study.
}

Table 1: The effect of first injection in the mean of hearing frequency improvement and speech discrimination score (SDS) between two groups.

\begin{tabular}{cccc}
\hline \multirow{2}{*}{ Frequency $(\mathrm{Hz})$} & \multicolumn{2}{c}{ Mean \pm Standard deviation } & \\
& Dexamethasone & Triamcinolone & p. value \\
\hline 500 & $58 \pm 6.5$ & $46.83 \pm 4.5$ & 127.0 \\
1000 & $57.1 \pm 3.7$ & $48.83 \pm 5.3$ & 235.0 \\
2000 & $56.66 \pm 4.2$ & $50.5 \pm 4.3$ & 424.0 \\
4000 & $56 \pm 5.5$ & $53.5 \pm 7.5$ & 742.0 \\
8000 & $58.66 \pm 3.5$ & $53.83 \pm 4.3$ & 480.0 \\
\hline
\end{tabular}




\begin{tabular}{cccc}
\hline SDS & $59.23 \pm 1.26$ & $69.66 \pm 4.2$ & 893.0 \\
\hline
\end{tabular}

Table 2: The effect of second injection in the mean of hearing frequency improvement and speech discrimination score (SDS) between two groups.

\begin{tabular}{cccc}
\hline \multirow{2}{*}{ Frequency $(\mathrm{Hz})$} & \multicolumn{2}{c}{ Mean \pm Standard deviation } & \multirow{2}{*}{ p. value } \\
& Dexamethasone & Triamcinolone & \\
\hline 500 & $51.5 \pm 10$ & $42.33 \pm 15$ & 268.0 \\
1000 & $53.33 \pm 3.7$ & $43.5 \pm 5.3$ & 214.0 \\
2000 & $52.66 \pm 5.4$ & $42.16 \pm 6.3$ & 136.0 \\
4000 & $51 \pm 5.5$ & $40.5 \pm 7.5$ & 479.0 \\
8000 & $55.16 \pm 3.5$ & $49.5 \pm 4.3$ & 450.0 \\
SDS & $57.96 \pm 11$ & $79.66 \pm 10$ & 325.0 \\
\hline
\end{tabular}

Table 3: The effect of third injection in the mean of hearing frequency improvement and speech discrimination score (SDS) between two groups.

\begin{tabular}{cccc}
\hline \multirow{2}{*}{ Frequency $(\mathbf{H z})$} & \multicolumn{2}{c}{ Mean \pm Standard deviation } & \\
\hline 500 & Dexamethasone & Triamcinolone & p. value \\
1000 & $47.66 \pm 22.1$ & $31.16 \pm 25$ & 039.0 \\
2000 & $46.33 \pm 10$ & $33.16 \pm 15$ & 069.0 \\
4000 & $46.66 \pm 10$ & $32.83 \pm 12$ & 066.0 \\
8000 & $46 \pm 18$ & $38.83 \pm 23$ & 406.0 \\
SDS & $46.16 \pm 12$ & $40.16 \pm 13$ & 603.0 \\
& $70.83 \pm 11$ & $79.70 \pm 10$ & 982.0 \\
\hline
\end{tabular}

Table 4: The effect of forth injection in the mean of hearing frequency improvement and speech discrimination score (SDS) between two groups.

\begin{tabular}{llll}
\hline \multirow{2}{*}{ Frequency $(\mathbf{H z})$} & \multicolumn{2}{c}{ Mean \pm Standard deviation } & \\
\hline 500 & Dexamethasone & Triamcinolone & p.value \\
1000 & $35.83 \pm 22$ & $27.66 \pm 25$ & 036.0 \\
2000 & $39 \pm 18$ & $25.33 \pm 23$ & 040.0 \\
4000 & $36.83 \pm 14$ & $25 \pm 16$ & 065.0 \\
8000 & $37.5 \pm 18$ & $30.33 \pm 23$ & 326.0 \\
SDS & $38.33 \pm 19$ & $30.66 \pm 23$ & 560.0 \\
\hline
\end{tabular}

Table 5: The effect of fifth injection in the mean of hearing frequency improvement and speech discrimination score (SDS) between two groups.

\begin{tabular}{cccc}
\hline \multirow{2}{*}{ Frequency $(\mathrm{Hz})$} & \multicolumn{2}{c}{ Mean \pm Standard deviation } & \\
& Dexamethasone & Triamcinolone & p. value \\
\hline 500 & $29.83 \pm 28$ & $16.33 \pm 30$ & 034.0 \\
1000 & $30.5 \pm 26$ & $18.16 \pm 30$ & 041.0 \\
2000 & $30.5 \pm 17$ & $18.66 \pm 16$ & 039.0 \\
4000 & $30.16 \pm 25$ & $22 \pm 31$ & 275.0 \\
8000 & $31.66 \pm 27$ & $22 \pm 31$ & 097.0 \\
SDS & $92.86 \pm 33$ & $95.65 \pm 25$ & 659.0 \\
\hline
\end{tabular}




\section{REFERENCES}

1. Kuhn M, Heman-Ackah SE, Shaikh JA, Roehm PC. Sudden sensorineural hearing loss: a review of diagnosis, treatment, and prognosis. Trends Amplif. 2011;15(3):91105.

2. Zahnert T. The differential diagnosis of hearing loss. Dtsch Arztebl Int. 2011;108(25):433-44.

3. Nosrati-Zarenoe R. Idiopathic Sudden Sensorineural Hearing Loss: Corticosteroid Treatment, the Diagnostic Protocol and Outcome [Doctoral thesis, comprehensive summary]. Linköping: Linköping University Electronic Press, 2011.

4. Hultcrantz E, Nosrati-Zarenoe R. Corticosteroid treatment of idiopathic sudden sensorineural hearing loss: analysis of an RCT and material drawn from the Swedish national database. European Archives of Oto-Rhino-Laryngology. 2015;272(11):3169-75.

5. Sung HK, Kang JC, Shin KH, An YS. Comparison of the Effects of Intratympanic Steroid Injection at Different Intervals in Sudden Sensorineural Hearing Loss. J Audiol Otol. 2020;24(1):24.

6. Khorsandi Ashtiani MT, Borgheie P, Yazdani N, Maghsoud $\mathrm{S}$. The effect of intratympanic dexamethasone with oral prednisolone as a primary treatment in idiopathic sudden sensorineural hearing loss. Iran $\mathrm{J}$ Otorhinolaryngol. 2012;24(66):19-22.

7. Berjis N, Soheilipour S, Musavi A, Hashemi SM. Intratympanic dexamethasone injection vs methylprednisolone for the treatment of refractory sudden sensorineural hearing loss. Adv Biomed Res. 2016;5:111-.

8. Rogha $M$, Abtahi $H$, Asadpour L, Ghazavi $H$, Pourmohammadi R, Maleki M, et al. Therapeutic Effect of Intratympanic Injection of Dexamethasone plus Hyaluronic Acid on Patients with Meniere's disease. Iran J Otorhinolaryngol. 2019;31(105):217-23.

9. Cinamon U, Bendet E, Kronenberg J. Steroids, carbogen or placebo for sudden hearing loss: a prospective double-blind study. European Archives of Oto-Rhino-Laryngology. $2001 ; 258(9): 477-80$.

10. Liu Rr, Ji-chuan C, Changyou J. Sudden sensorineural hearing loss treated with intratympanic dexamethasone injection. Journal of Otology. 2010;5(1):4-7.

11. Lee KH, Ryu SH, Lee HM, Park SK, Kim H-J, Chang J. Is Intratympanic Dexamathasone Injection Effective for the Treatment of Idiopathic Sudden Sensorineural Hearing Loss? J Audiol Otol. 2015;19(3):154-8.

12. Wang Y-w, Ren J-h, Lu Y-d, Yin T-f, Xie D-h. Evaluation of intratympanic dexamethasone for treatment of refractory sudden sensorineural hearing loss. Journal of Zhejiang University SCIENCE B. 2012;13(3):203-8.

13. Berjis N, Soheilipour S, Musavi A, Hashemi SM. Intratympanic dexamethasone injection vs methylprednisolone for the treatment of refractory sudden sensorineural hearing loss. Advanced biomedical research. 2016;5

14. Choung $\mathrm{YH}$, Park K, Shin YR, Cho MJ. Intratympanic dexamethasone injection for refractory sudden sensorineural hearing loss. The Laryngoscope. 2006;116(5):747-52.

15. Gloddek B, Lamm K, Arnold W. Pharmacological influence on inner ear endothelial cells in relation to the pathogenesis of sensorineural hearing loss. ADVANCES IN OTORHINOLARYNGOLOGY. 2002;59:75-83.

16. Tabuchi K, Oikawa K, Uemaetomari I, Tsuji S, Wada T, Hara A. Glucocorticoids and dehydroepiandrosterone sulfate ameliorate ischemia-induced injury of the cochlea. Hearing research. 2003;180(1, 2):51-6.
17. Xenellis J, Papadimitriou N, Nikolopoulos T, Maragoudakis P, Segas J, Tzagaroulakis A, et al. Intratympanic steroid treatment in idiopathic sudden sensorineural hearing loss: a control study. Otolaryngology-Head and Neck Surgery. 2006;134(6):940-5.

18. Plontke SK, Löwenheim $\mathrm{H}$, Mertens J, Engel C, Meisner $\mathrm{C}$, Weidner A, et al. Randomized, double blind, placebo controlled trial on the safety and efficacy of continuous intratympanic dexamethasone delivered via a round window catheter for severe to profound sudden idiopathic sensorineural hearing loss after failure of systemic therapy. The Laryngoscope. 2009;119(2):359-69.

19. Ho Ahn J, Woul Han M, Heui Kim J, Woo Chung J, Hyun Yoon T. Therapeutic effectiveness over time of intratympanic dexamethasone as salvage treatment of sudden deafness. Acta oto-laryngologica. 2008;128(2):12831.

20. Kiliç R, Safak MA, Oguz H, Kargin S, Demirci M, Samim E, et al. Intratympanic methylprednisolone for sudden sensorineural hearing loss. Otology \& Neurotology. $2007 ; 28(3): 312-6$.

21. Dahm V, Nieratschker M, Riss D, Kaider A, Auinger A, Honeder $\mathrm{C}$, et al. Intratympanic triamcinolone acetonide as treatment option for idiopathic sudden sensorineural hearing loss. Otology \& Neurotology. 2019;40(6):720-7.

22. Ferri $E$, Frisina $A$, Fasson AC, Armato $E$, Spinato G, Amadori M. Intratympanic steroid treatment for idiopathic sudden sensorineural hearing loss after failure of intravenous therapy. ISRN otolaryngology. 2012;2012.

23. Seggas I, Koltsidopoulos P, Bibas A, Tzonou A, Sismanis A. Intratympanic steroid therapy for sudden hearing loss: a review of the literature. Otology \& Neurotology. 2011;32(1):29-35

24. Bird PA, Begg EJ, Zhang M, Keast AT, Murray DP, Balkany TJ. Intratympanic versus intravenous delivery of methylprednisolone to cochlear perilymph. Otology \& Neurotology. 2007;28(8):1124-30.

25. Suzuki H, Wakasugi $T$, Kitamura $T$, Koizumi $H$, Do BH, Ohbuchi T. Comparison of 2 and 4 intratympanic steroid injections in the treatment of idiopathic sudden sensorineural hearing loss. Annals of Otology, Rhinology \& Laryngology. 2018;127(4):235-40.

26. Ahn JH, Yoo MH, Yoon TH, Chung JW. Can intratympanic dexamethasone added to systemic steroids improve hearing outcome in patients with sudden deafness? The Laryngoscope. 2008;118(2):279-82.

27. Ashtiani MK, Firouzi F, Bastaninejad S, Dabiri S, Nasirmohtaram S, Saeedi N, et al. Efficacy of systemic and intratympanic corticosteroid combination therapy versus intratympanic or systemic therapy in patients with idiopathic sudden sensorineural hearing loss: a randomized controlled trial. European Archives of Oto-Rhino-Laryngology. 2018;275(1):89-97. 